\title{
Parásitos intestinales en monos congo Alouatta palliata (Primates: Cebidae) de Costa Rica
}

\author{
Misael Chinchilla Carmona ${ }^{1,3}$, Olga Guerrero Bermúdez ${ }^{1}$, Gustavo A. Gutiérrez-Espeleta², \\ Ronald Sánchez Porras ${ }^{4} \&$ Beatriz Rodríguez Ortiz ${ }^{1}$ \\ 1 Centro de Investigación en Enfermedades Tropicales, Departamento de Parasitología, Facultad de Microbiología, \\ Universidad de Costa Rica. Chinchillacm@UCIMED.com \\ 2 Escuela de Biología, Universidad de Costa Rica. \\ 3 Departamento de Investigación, Universidad de Ciencias Medicas (UCIMED). \\ 4 Sede de Occidente, Universidad de Costa Rica. \\ Correos electrónicos: (G. Gutiérrez) ggutier@biologia.ucr.ac.cr; (O. Guerrero) abriceno@racsa.co.cr; (R. Sánchez) \\ rsanchez@cariari.ucr.ac.cr \\ Correspondencia: M. Chinchilla: chinchillacm@ucimed.com
}

$$
\text { Recibido 13-IV-2005. Corregido 08-IX-2005. Aceptado 10-IX-2005. }
$$

\begin{abstract}
Intestinal parasites in howler monkeys Alouatta palliata (Primates: Cebidae) of Costa Rica. Fecal samples of 102 howler monkeys (Alouatta palliata) from several sites of Costa Rica were studied for intestinal parasites. The zones studied were: Central Valley (San Ramón, Alajuela), Central Pacific (Chomes and Manuel Antonio National Park, Puntarenas), North Pacific (Palo Verde Park and Playa Potrero, Guanacaste), Chira Island in the Nicoya Gulf and Caribean area (Cahuita, Limón). Animals were anesthetized with dards containing Telazol in order to collect the fecal material; some monkeys defecated spontaneously and others by direct stimulation. Samples were studied in saline solution $(0.85 \%)$ and a Iodine solution, or stained with Haematoxylin. The material was also cultured in Dobell culture medium to determine the presence of amoeba and flagellates. Strongyloides, Controrchis, Trypanoxyuris genera were found in $3.4 \%$ of the samples. In addition $16.7 \%$ to $80 \%$ of the animals showed protozoa infection with Endolimax, Entamoeba, Trichomonas and Giardia. It is discussed the relationships of parasite infection with environmental conditions, animal population and human presence, specially in the monkey conservation programs point of view. Rev. Biol. Trop. 53(3-4): 437-445. Epub 2005 Oct 3.
\end{abstract}

Key words: Intestinal parasites, howler monkeys, Alouatta palliata, Cebidae, Primates, Costa Rica.

El riesgo de extinción de primates en el mundo es un hecho ampliamente conocido en todo el mundo, ya que las especies de este grupo de animales, al igual que las de otros grupos se encuentra en evidente peligro de desaparecer.

En Costa Rica, como en muchos de los países mencionados en ese informe, existe una tendencia a la disminución en el número de individuos y especies de animales silvestres, en las que se incluyen los monos con que cuenta este país a saber: mono congo o aullador (Alouatta palliata Gray, 1849), carablanca (Cebus capucinus, Linnaeus, 1758), tití o ardilla (Saimiri oerstedii, Reinhardt, 1872) y colorado o araña (Ateles geoffroyi Kuhl, 1820) (Villalobos et al. 2004, Zaldívar et al. 2004). La creación de áreas protegidas ha contribuido a la conservación de estas especies, mientras que la destrucción del habitat y la cacería clandestina, son casi siempre los factores responsables de la disminución del número de individuos en las poblaciones. Las infecciones parasitarias podrían ser también causa de este fenómeno, especialmente si se toma en cuenta que cada día existe mayor contacto entre estos primates y el ser humano, quien como sabemos, es infectado por varios parásitos capaces de producir 
problemas patológicos importantes. Al respecto se conocen los informes de la presencia de amebas y flagelados (Gozalo y Tantalean 1996) en las 4 especies de monos estudiados en todo el proyecto del que es parte este informe y que lleva alrededor de tres años de trabajo.

Específicamente en el caso del género Alouatta, se cuenta con hallazgos importantes en ejemplares provenientes de otros países del Continente Americano (Stuart et al. 1998). Se han reportado protozoarios de los géneros Entamoeba, Giardia, Trichomonas, Chilomastix, Endolimax y Balantidium. En cuanto a helmintos se refiere, se cuenta con un estudio realizado en Costa Rica en A. palliata del Centro Ecológico La Pacífica, Guanacaste (Stuart et al. 1990) en que se informa de la presencia de un estrongilideo y un tremátodo, Controrchis caballeroi, Controrchis biliophilus (C. caballeroi) (Jiménez-Quirós y Brenes 1957-1958, Rodríguez-Ortiz et al. 2004). En otro estudio realizado en la Reserva Biológica La Selva (Stoner 1996)se encontraron algunos helmintos en las heces de estos primates, pero no se informó de protozoarios intestinales.

Este estudio pretende analizar la fauna parasitaria intestinal que podría representar algún impacto negativo en la salud de los monos congo y eventualmente justificar el deterioro de las poblaciones. Para lo anterior se hizo un estudio de las heces de 102 monos de la especie $A$. palliata provenientes de diversos lugares de Costa Rica.

\section{MATERIAL Y MÉTODOS}

Se estudiaron 99 ejemplares de la especie A. palliata provenientes de diversos lugares de Costa Rica (Fig. 1): 5 del Valle Central (San Ramón, Alajuela), 14 del Pacífico Central (Chomes y Parque Manuel Antonio, Puntarenas), 35 del Pacífico Norte (Parque Nacional Palo Verde, Gran Nicoya y Playa Potrero, Guanacaste), 5 de la Isla Chira situada en el Golfo de Nicoya y 29 de la región del Caribe (Cahuita, Limón). Para la captura de los monos en su estado silvestre, se utilizaron dardos con compuestos anestésicos, mediante técnicas estándares (Glander et al. 1991). El anestésico general empleado fue el hidrocloruro de tiletamina, un análogo de la ketamina, disponible en una combinación 1:1 con zolazepam (una pirazolodiazepina) conocido comercialmente como Zoletil®. El zolazepam al estar mezclado con la tiletamina genera un aumento en sus efectos anestésicos, ayuda a la relajación muscular, disminuye las posibles convulsiones y establece una recuperación paulatina (Swan 1992). La dosis recomendada de este compuesto para primates es de 5-10 $\mathrm{mg} / \mathrm{kg}$ (IACUC 1998). Los animales fueron recibidos al caer anestesiados en una red sostenida por 4 personas.

Luego fueron pesados y se les realizó el examen físico, después de lo cual defecaron o fueron estimulados para hacerlo por medio de ligera presión en el abdomen. Las heces fueron

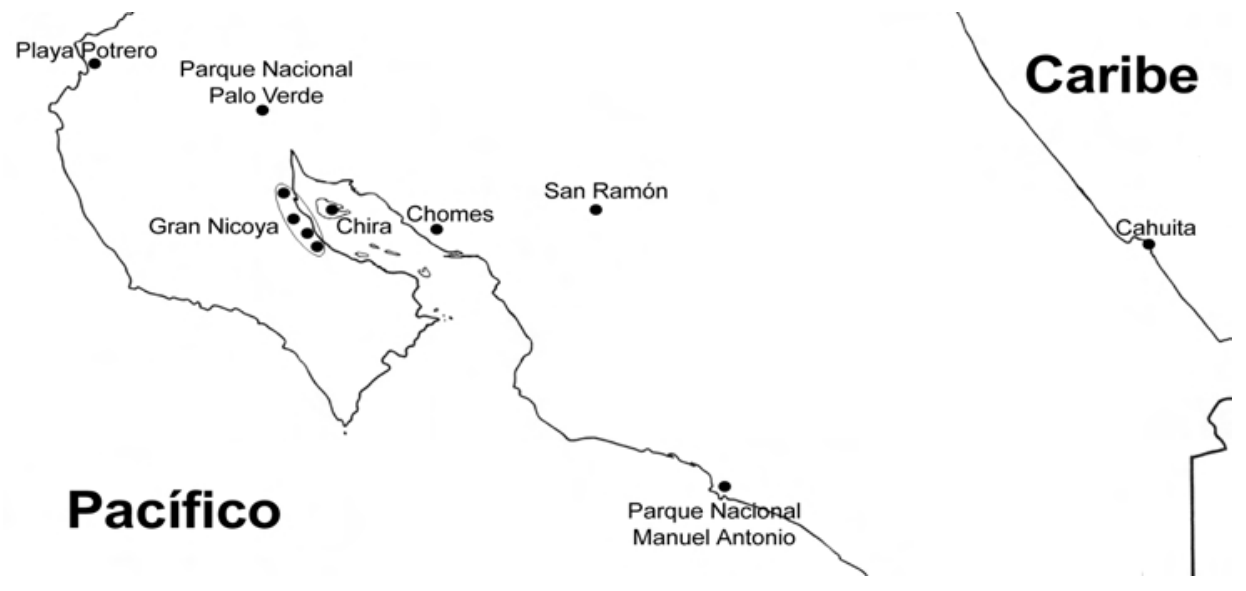

Fig. 1. Zonas de estudio de parásitos en monos de la especie A.palliata. 
colectadas en recipientes plásticos para transportarlas al laboratorio. Tres de las muestras, 2 de San Ramón y 1 del Parque Manuel Antonio, totalmente individualizadas pero sin identificación del animal de origen, fueron incluidas dentro del estudio (Cuadros 3 y 4) por el interés parasitológico que conlleva. Por supuesto que estas muestras no son tomadas en cuenta en los estudios relacionados con sexo y peso de los monos (Cuadros 5 y 6).

Después de realizar todos los exámenes antes indicados, generalmente ellos comenzaban a despertar, pero de todas maneras por medio de aplicación vía oral de pequeñas cantidades de agua se aceleraba el proceso. Los animales fueron vigilados hasta que presentaran la capacidad de asirse con facilidad a los objetos, momento en el que se les colocaba en un árbol de fácil acceso para que ascendiera hacia otros árboles de mayor altura.

El material se estudió en forma directa, suspendiéndolo en solución salina al $0.85 \%$ o en una solución de lugol D’ Antoni para determinar la presencia de trofozoitos o quistes de protozoarios, así como la de huevecillos, larvas o adultos de helmintos intestinales. Parte de la muestra se sembró en el medio de Dobell modificado para determinar el crecimiento de flagelados los cuales fueron diagnosticados por su crecimiento y motilidad. Los otros organismos fueron identificados por su morfología, observada tanto en forma directa como en preparaciones teñidas con hematoxilina férrica de Heidenhain, donde se analizó su estructura nuclear, tipo de cromatoidales de los quistes e inclusiones citoplasmáticas de los trofozoitos. En el caso de Entamoeba chattoni, fue necesario efectuar mediciones y otras observaciones morfológicas con base en la descripción original (Burrows 1955) dada la similitud con las especies de amebas conocidas.

Se trató de establecer la relación entre las especies y el número de parásitos encontrados en los primates y cercanía de éstos con el ser humano, para lo cual se tomó en cuenta el área y la población de las regiones en estudio (Cuadro 1). También se relacionaron los resultados con las condiciones climáticas prevalecientes en las regiones estudiadas (Cuadro 2).

\section{RESULTADOS}

El 44\% de las muestras fueron positivas por protozoarios (Cuadro 3) tales como: Entamoeba histolytica/Entamoeba dispar, Entamoeba coli y Entamoeba hartmanni, parásitas también del ser humano, así como Entamoeba chattoni, característica de monos. Los animales de las zonas de Isla Chira, Playa Potrero, San Ramón

CUADRO 1

Población humana en las zonas geográficas en donde se colectaron las muestras de la especie A. palliata

\begin{tabular}{lccc}
\multicolumn{1}{c}{ Zona Geográfica } & Area $\left(\mathrm{Km}^{2}\right)$ & Población & Habit/Km ${ }^{2 *}$ \\
Chomes & 127.6 & 4162 & 33 \\
Chira (Manzanillo) & 101.86 & 3180 & 31 \\
Manuel Antonio & 6.82 & 5000 & 733 \\
Quepos & 222.89 & 14948 & 67 \\
Cahuita & 173.41 & 5426 & 31 \\
San Ramón - Centro & 1.29 & 9697 & 7517 \\
Potrero - Sardinal & 240.45 & 10204 & 43 \\
Gran Nicoya - Sn. Antonio & 338.17 & 6716 & 20 \\
Palo Verde & 57.04 & 2114 & 37
\end{tabular}

* Número aproximado de habitantes por Km

Fuente: Documentación en Atlas de Costa Rica. 2000- Origen Geotecnologías S.A. Según censo del 2000. 


\section{CUADRO 2}

Condiciones climáticas en los lugares de captura en el presente estudio indicando temperatura, precipitación, zona de vida y caracteristicas de los bosques que la conforman

Lugar captura

Chomes
Parque Nacional
Palo Verde
Parque Nacional
Cahuita
Chira

San Ramón

Gran Nicoya

Playa Potrero

Parque Nacional Manuel Antonio

$$
\begin{aligned}
& \text { Temp. media anual } \\
& \text { (periodo seco) }
\end{aligned}
$$

$24^{\circ} \mathrm{C}$ a $27.8^{\circ} \mathrm{C}$ (Periodo seco efectivo de 3.5 a 5 meses)

$24^{\circ} \mathrm{C}$ a $27.8^{\circ} \mathrm{C}$ (Periodo seco efectivo de 3.5 a 5 meses)

$24^{\circ} \mathrm{C}$ a $27^{\circ} \mathrm{C}$ (Periodo seco variable de 0 a 5 meses)

$24^{\circ} \mathrm{C}$ a $27.8^{\circ} \mathrm{C}$ (Periodo seco consecutivo es de 6.5 meses)

$17^{\circ} \mathrm{C}$ a $24^{\circ} \mathrm{C}$ (Periodo seco efectivo de 0 a 5 meses)

$24^{\circ} \mathrm{C}$ a $27.8^{\circ} \mathrm{C}$ (Periodo seco efectivo de 3.5 a 5 meses)

$24^{\circ} \mathrm{C}$ a $27.8^{\circ} \mathrm{C}$ (Periodo seco efectivo es de 6.5 meses)

$24^{\circ} \mathrm{C}$ a $27^{\circ} \mathrm{C}$ (Periodo seco efectivo de 0 a 5 meses)
Precipitación

$1500 \mathrm{~mm}$ a $1950 \mathrm{~mm}$

$1500 \mathrm{~mm}$ a $1950 \mathrm{~mm}$

$1950 \mathrm{~mm}$ a $3000 \mathrm{~mm}$

$2000 \mathrm{~mm}$ a $4000 \mathrm{~mm}$

$1100 \mathrm{~mm}$ a $1500 \mathrm{~mm}$

$1500 \mathrm{~mm}$ a $1950 \mathrm{~mm}$

$1100 \mathrm{~mm}$ a $1500 \mathrm{~mm}$

$3000 \mathrm{~mm}$ a $4000 \mathrm{~mm}$
Tipo de bosque

Bosque húmedo premontano transición a basal tropical (Bh-P)

(Bh-P)

Bosque húmedo tropical (Bh-T)

Bosque seco tropical (Bs-T)

Bosque muy húmedo Premontano (Bmh-P)

(Bh-P)

(Bs-T)

(Bmh-P)

Fuente: Instituto metereológico Nacional. www.iied.guias.costa rica.com/cr3.htm

\section{CUADRO 3}

\begin{tabular}{|c|c|c|c|c|c|c|c|c|c|}
\hline \multirow{3}{*}{$\begin{array}{c}\text { Zona } \\
\text { Geográfica }\end{array}$} & \multirow{3}{*}{$\begin{array}{c}\mathrm{N}^{\circ} \mathrm{de} \\
\text { Muestras }\end{array}$} & \multicolumn{6}{|c|}{ Protozoos } & \multirow{2}{*}{\multicolumn{2}{|c|}{$\begin{array}{c}\text { Total positivos } \\
\text { (Al menos } 1 \text { parásito) }\end{array}$}} \\
\hline & & \multicolumn{2}{|c|}{ Amebas * } & \multicolumn{2}{|c|}{ Trichomonas } & \multicolumn{2}{|c|}{ Giardia } & & \\
\hline & & $\mathrm{N}^{\circ}$ & $\%$ & $\mathrm{~N}^{\circ}$ & $\%$ & $\mathrm{~N}^{\circ}$ & $\%$ & $\mathrm{~N}^{\circ}$ & $\%$ \\
\hline Chomes & 9 & 6 & 66.7 & 1 & 11.1 & 0 & 0 & 6 & 66.7 \\
\hline Palo Verde & 20 & 3 & 15.0 & 1 & 5.0 & 1 & 5.0 & 5 & 25.0 \\
\hline Cahuita & 29 & 9 & 31.0 & 1 & 3.4 & 1 & 3.4 & 11 & 37.9 \\
\hline Chira & 5 & 4 & 80.0 & 0 & 0 & 0 & 0 & 4 & 80.0 \\
\hline San Ramón & 7 & 4 & 57.1 & 0 & 0 & 1 & 14.3 & 5 & 71.4 \\
\hline Gran Nicoya & 18 & 7 & 38.9 & 0 & 0 & 0 & 0 & 7 & 38.9 \\
\hline Playa Potrero & 8 & 6 & 75.0 & 0 & 0 & 5 & 62.5 & 6 & 75.0 \\
\hline Manuel Antonio & 6 & 1 & 16.7 & 0 & 0 & 0 & 0 & 1 & 16.7 \\
\hline TOTALES & 102 & 40 & 39.2 & 3 & 2.9 & 8 & 7.8 & 45 & 44.1 \\
\hline
\end{tabular}

Presencia de protozoos en 102 muestras de heces de monos de la especie Alouatta palliata de 8 zonas geográficas

Se incluyen 2 muestras de heces de monos de San Ramón y 1 de monos de Manuel Antonio cuyo origen animal no se conoce.

* Géneros Entamoeba y Endolimax 
y Chomes presentaron los mayores porcentajes de infección por protozoarios $(80 \%, 75 \%$, $71.4 \%$ y 66.7 respectivamente).

La infección por helmintos fue porcentualmente más baja (Cuadro 4), con excepción de los animales de la Isla de Chira en donde casi todos los individuos estudiados estaban positivos. La infección por sexo de los monos congo fue similar (Cuadro 5).

Al agrupar los animales estudiados como infantes, juveniles y adultos, los porcentajes de infección por al menos un parásito fueron de $81.8 \%, 52.6 \%$ y $49.3 \%$ respectivamente. El análisis del ámbito de pesos y la presencia de parásitos indicó un ligero aumento en el grado de infección en animales de menos peso $(0.25$ a $5 \mathrm{Kg}$ ). No se toma en cuenta como parámetro comparativo los datos de monos con pesos mayores a $10 \mathrm{Kg}$ dado el poco número de ejemplares con tal condición (Cuadro 6).

Las poblaciones de monos aulladores se desplazan muy cerca de las viviendas en las zonas estudiadas, sin importar, aparentemente, la densidad de la población humana (Cuadro 1).

CUADRO 4

Presencia de helmintos en 102 muestras de heces de monos de la especie Alouatta palliata de 8 zonas geográficas

\begin{tabular}{|c|c|c|c|c|c|c|c|c|c|}
\hline \multirow{2}{*}{$\begin{array}{c}\text { Zona } \\
\text { Geográfica }\end{array}$} & \multirow{2}{*}{$\begin{array}{c}\mathrm{N}^{\circ} \mathrm{de} \\
\text { Muestras }\end{array}$} & \multicolumn{6}{|c|}{ Helmintos } & \multicolumn{2}{|c|}{ TOTAL } \\
\hline & & $\mathrm{N}^{\circ}$ & $\%$ & $\mathrm{~N}^{\circ}$ & $\%$ & $\mathrm{~N}^{\circ}$ & $\%$ & $\mathrm{~N}^{\circ}$ & $\%$ \\
\hline Chomes & 9 & 1 & 11.1 & 2 & 22.2 & 0 & 0 & 3 & 33.3 \\
\hline Palo Verde & 20 & 0 & 0 & 1 & 5 & 0 & 0 & 1 & 5 \\
\hline Cahuita & 29 & 1 & 3.4 & 0 & 0 & 0 & 0 & 1 & 3.4 \\
\hline Chira & 5 & 3 & 60 & 0 & 0 & 1 & 20 & 4 & 80 \\
\hline San Ramón & 7 & 0 & 0 & 1 & 14.3 & 0 & 0 & 1 & 14.3 \\
\hline Gran Nicoya & 18 & 1 & 5.6 & 3 & 16.7 & 1 & 5.6 & 5 & 27.8 \\
\hline Playa Potrero & 8 & 0 & 0 & 2 & 25 & 0 & 0 & 2 & 25 \\
\hline Manuel Antonio & 6 & 0 & 0 & 1 & 16.7 & 0 & 0 & 1 & 16.7 \\
\hline TOTAL & 102 & 6 & 5.9 & 10 & 9.8 & 2 & 2.0 & 18 & 17.6 \\
\hline
\end{tabular}

Se incluyen 2 muestras de heces de monos de San Ramón y 1 de monos de Manuel Antonio de origen animal desconocido

CUADRO 5

Relación entre el porcentaje de positividad por parásitos y el sexo de monos de la especie Alouatta palliata

\begin{tabular}{|c|c|c|c|c|c|c|c|c|c|c|}
\hline \multirow{3}{*}{ Sexo } & & & \multicolumn{8}{|c|}{ Positividad } \\
\hline & \multicolumn{2}{|c|}{ Negativos } & \multicolumn{2}{|c|}{ Total } & \multicolumn{2}{|c|}{ Protozoos } & \multicolumn{2}{|c|}{ Helmintos } & \multicolumn{2}{|c|}{ Ambos } \\
\hline & $\mathrm{N}^{\circ}$ & $\%$ & $\mathrm{~N}^{\circ}$ & $\%$ & $\mathrm{~N}^{\circ}$ & $\%$ & $\mathrm{~N}^{\circ}$ & $\%$ & $\mathrm{~N}^{\circ}$ & $\%$ \\
\hline Hembras & 24 & 24.2 & 24 & 24.2 & 15 & 15.1 & 5 & 5.0 & 3 & 3.0 \\
\hline Machos & 24 & 24.2 & 27 & 27.3 & 20 & 20.2 & 3 & 5.0 & 5 & 5.0 \\
\hline Total & 48 & 48.5 & 51 & 51.5 & 35 & 35.4 & 8 & 8.0 & 8 & 8.0 \\
\hline
\end{tabular}


CUADRO 6

Porcentaje de positividad por parásitos en relación con ámbitos de peso de los 99 monos estudiados

\begin{tabular}{|c|c|c|c|c|c|c|c|c|c|}
\hline \multirow{3}{*}{ Sexo } & \multicolumn{9}{|c|}{ Ambitos de peso $(\mathrm{Kg})$} \\
\hline & \multicolumn{3}{|c|}{0.25 a 5} & \multicolumn{3}{|c|}{5.1 a 10} & \multicolumn{3}{|c|}{10.1 a 15.8} \\
\hline & Total & Pos* & $\%$ & Total & Pos* & $\%$ & Total & Pos* & $\%$ \\
\hline Hembras & 28 & 16 & 57.1 & 22 & 7 & 31.8 & 0 & 0 & 0 \\
\hline Machos & 11 & 3 & 27.3 & 34 & 18 & 52.9 & 4 & 3 & 75 \\
\hline Total & 39 & 19 & 48.7 & 56 & 25 & 44.6 & 4 & 3 & 75 \\
\hline
\end{tabular}

\section{DISCUSIÓN}

Stuart et al. (1990, 1998) mencionan en su revisión sobre parásitos de primates, la presencia de protozoarios intestinales de los géneros Entamoeba, Giardia, Retortamonas, Trichomonas, Chilomastix y Balantidium en monos del género Alouatta de Brazil y Guayana Francesa. Específicamente en la especie A. palliata dichos autores informan los mismos protozoarios en Panamá y México. La presencia de helmintos intestinales de los géneros Ascaris, Ancylostoma y Trypanoxyuris ha sido documentada en Brazil, Colombia y Surinam. Específicamente en Costa Rica, se menciona la presencia de estrongilidios y helmintos del género Trypanoxyuris en A. palliata (Stuart et al. 1988).

El hallazgo de E. chattoni, es importante ya que constituye el primer informe de este parásito en Centro América. Además la presencia de organismos similares a E. histolytica/E. dispar y $G$. intestinalis, especies patógenas para el ser humano, podría ser un factor negativo en la supervivencia de esos animales.

Este es el primer informe en el cual se analiza un número importante de animales y se observa una infección notoria por parásitos intestinales (Cuadros 3 y 4). Se observó que la infección por protozoarios es más elevada que por helmintos. Esto se debe muy probablemente a que dentro de los parásitos intestinales los ciclos de vida de los helmintos son, en general, más complejos que en los protozoarios, requiriendo algunas veces condiciones especiales del ambiente para que se desarrolle la forma infectante.

La importancia de los parásitos como un factor que afecta la densidad y distribución de las especies de monos, así como en la conservación biológica, ha sido reconocida por varios autores (Anderson 1979, May 1988, Scott 1988). Además la llamada Medicina de conservación indica que "las enfermedades juegan un papel muy importante en la dinámica de poblaciones" y que el contacto cercano entre animales y humanos amenaza la salud y la conservación (Daszak et al. 2000). Por otro lado, el proceso de fragmentación boscosa ha hecho que los animales vayan perdiendo espacio, aproximándose cada vez más a la población humana y variando su dieta tradicional (Gilbert y Dodds 1987). Esto produce un mayor riesgo de transmisión de parásitos de humanos hacia los monos como se observa en este estudio, especialmente en el caso de los protozoarios. Esto se comprueba al observar que las muestras de Chomes, Isla de Chira, San Ramón y Playa Potrero fueron las que presentaron mayores porcentajes de infección $(66.7 \%, 80 \%, 71.4 \%$ y 75\% respectivamente) (Cuadro 3). En los cuatro casos los animales estaban mucho más cercanos a la población o inclusive limitados a un espacio muy pequeño como en la Isla de Chira y San Ramón, este último con una densa población humana (Cuadro 1). El caso del parque nacional Manuel Antonio parece salirse del formato, pues es visitado por gran 
cantidad de turistas que en cierto modo establecen mayor presencia del ser humano con los monos. Sin embargo, la existencia de una área boscosa importante en esta zona y la posibilidad de que algún componente propio de la dieta de los animales pueda ejercer algún efecto antiparasitario, podrían ser las razones de tal incongruencia. Los índices de infección en los animales de las diferentes regiones estudiadas no dependen aparentemente de las condiciones climáticas de éstas (Cuadro 2), ya que igualmente se dan infecciones altas de parásitos tanto en bosque seco (Chira) como en bosque húmedo (Chomes y San Ramón).

En este trabajo los animales con un peso de 0.25 a $5 \mathrm{Kg}$ presentaron un porcentaje de infección mayor que aquellos con un peso de 5.1 a $10 \mathrm{Kg}$ (Cuadro 6). Otros autores (Stuart et al. $1990,1998)$ han indicado que las infecciones son más frecuentes en adultos de más edad (de más peso). En nuestro estudio se encontró un porcentaje mayor de infecciones en primates de más de $10 \mathrm{Kg}$ pero dado el poco número de animales con este peso no es posible obtener conclusiones válidas. Analizando los animales de acuerdo con su categoría de infantes, juveniles y adultos, observamos que el mayor porcentaje de infección (81.8\%), estuvo presente en los infantes lo cual semeja a lo que ocurre con las infecciones parasitarias humanas en que los niños son mayormente proclives a las infecciones.

Otro aspecto a tomar en cuenta es la fragmentación boscosa en Costa Rica, ya que induce a una mayor concentración de las tropas de estos animales en un menor espacio. Al respecto (Nunn et al. 2003) estudiaron al menos cuatro factores como problemas determinantes del aumento de especies parasitarias en los monos y concluyeron que la densidad poblacional de primates era el factor más importante, no sólo por la mayor presencia, sino también por la más amplia variación de especies de agentes infecciosos.

El conocimiento de infecciones parasitarias en estos animales, induce a pensar en la importancia del desarrollo de lo que algunos países como Colombia, denominan la medicina de la conservación (Nassar-Montoya et al. 2001) en donde se ha desarrollado una gran infraestructura y logística, tendiente a proteger estos animales a través del tratamiento de sus problemas tanto de carácter infeccioso como metabólicos.

En Costa Rica al igual que en otros países, la implementación de programas similares de vigilancia sostenida de la salud de la fauna silvestre, podrían ser de gran importancia para proteger los monos aulladores, cuya presencia es vital para mantener el equilibrio biológico de los bosques de donde son nativos. Aparte de este valor en la naturaleza, está el aspecto económico agregado que se brinda a las áreas que habitan, incrementando su potencial turístico.

El hallazgo de las infecciones parasitarias aquí informadas, podría ser entonces un aspecto importante a tomar en cuenta, especialmente al establecer programas de conservación de los pocos monos que todavía quedan en Costa Rica.

\section{AGRADECIMIENTOS}

Este trabajo fue financiado en parte con el proyecto No. 111-A1-015 de la Vicerrectoría de Investigación de la Universidad de Costa Rica y por el Departamento de Investigación de la Universidad de Ciencias Médicas (UCIMED). Los autores agradecen profundamente a los estudiantes de grado y posgrado de Biología y Microbiología de la Universidad de Costa Rica, Universidad Nacional e Instituto Tecnológico por su apoyo en la captura y manejo de los animales. También agradecemos a Mario Vargas V. y José M. Mora por la revisión del manuscrito y externar sus valiosos consejos para mejorarlo.

\section{RESUMEN}

Se estudiaron las heces de 102 monos de la especie Alouatta palliata (monos aulladores o congos) de las siguientes regiones de Costa Rica. Valle Central: San Ramón (Alajuela); Pacífico Central: Isla Chira y Parque Manuel Antonio (Puntarenas); Pacífico Norte: Palo Verde, Playa Potrero, Nicoya (Guanacaste) y Caribe: Cahuita 
(Limón). Los animales capturados fueron anestesiados con dardos conteniendo Telazol®. La mayoría defecaron espontáneamente o se les estimulaba a defecar. Las muestras se estudiaron en el laboratorio en forma directa en solución salina $(0.85 \%)$ y una solución de yodo o teñidas con hematoxilina para identificar los parásitos correspondientes. El material fue cultivado en el medio de Dobell para determinar la presencia de amebas y flagelados. Se encontraron helmintos de los géneros Trypanoxyuris, Strongyloides y Controrchis y protozoarios de los géneros Endolimax, Entamoeba, Trichomonas y Giardia. Los porcentajes de infección por protozoarios oscilaron entre $16.7 \%$ y $80 \%$ y se encontraron helmintos en un $3.4 \%$ a un $80 \%$ de los animales de acuerdo con la zona estudiada. No se encontraron diferencias importantes de parasitosis relacionadas con el sexo pero si existió mayor infección en los animales más jóvenes. Se establece la relación entre los índices de infección y algunos parámetros tales como las áreas estudiadas, el peso de los animales y el contacto con el ser humano.

Palabras clave: Parasitos intestinales, monos congo, Alouatta palliata, Cebidae, Primates, Costa Rica.

\section{REFERENCIAS}

Anderson, R.M. 1979. Parasite pathogenicity and the depression of host population equilibria. Nature 279: 150-152.

Burrows, R.B. 1955. Endamoeba polecki infections in man. Am. J. Hyg. 162: 156-157.

Clarke, M.R., C.M. Crockett, E.L. Zucker \& M.E. ZandícarRuiz. 2002. Mantled howler population of Hacienda La Pacífica, Costa Rica, between 1991 and 1998: Effects of deforestation. Am. J. Primatology 56: 155-163.

Clarke, M.R., D.A. Collins \& E.L. Zucker. 2002. Responses to deforestation in a group of mantled howlers (Alouatta palliata) in Costa Rica. Int. J. Primatology. 23: 365-381.

Daszak, P., A.A. Cunningham \& A.D. Hyatt. 2000. Emerging infectious diseases of wildlife-treats to biodiversity and human health. Science 287: 443-449.

IACUC, 1998. IACUC guidelines for the care and use of live vertebrate animals. Institutional Animal Care and Use Committee. Appendix F. (También disponible en línea: http://WWW.unmc.edu/Education/Animal/ guide/iacuc-GUIDELINES.pdf )

Gilbert, F.F. \& D.G. Dodds. 1978. The philosophy and practice of wildlife management. Robert E. Krieger, Malabar, Florida, EEUU.

Glander, K.E. L..M. Fedigan, L. Fedigan \& C. Chapman. 1991. Field Methods for Capture and Measurement of
Three Monkey Species in Costa Rica. Fol. Primatol. 57: 70-82.

Gozalo, A.C. \& M. Tantaleán. 1996. Parasitic Protozoa in Neotropical primates. Lab. Primate Newsletter 35: $1-7$.

Jiménez-Quirós, O. \& R. Brenes. 1957. Helmintos de la República de Costa Rica. V. Sobre la validez del género de Controrchis Price, 1928 (Trematoda, Dicrocoeliidae) y descripción de Controrchis caballeroi n. sp. Rev. Biol. Trop. 5: 103-121.

Jiménez-Quirós, O \& R. Brenes. 1958. Nota sobre el tremátodo Controrchis caballeroi Jiménez y Brenes, 1957. Rev. Biol. Trop. 6: 189-190.

May, R.M. 1988. Conservation and Disease. Conserv. Biol. 2: 28-30.

Nassar, E., V. Pereira \& T. Vodovoz. 2003. Medicina de la conservación en el estudio de poblaciones naturales de primates en Colombia. Primatol. Nuevo Mundo 2003: 239-252.

Nunn, C.L., S. Altizer, K.E. Jones \& W. Sechrest. 2003. Comparative Tests of parasite species richness in Primates. Amer. Nat. 162: 597-614.

Rodríguez-Ortíz, B., L. García-Prieto, \& G. Pérez-Ponce de León. 2004. Checklist of the helminth parasites of vertebrates in Costa Rica. Rev. Biol. Trop. 52: 313-354.

Sargeaunt, P.G., J.E. Williams \& D.M. Jones. 1982. Electrophoretic Isoenzyme patterns of Entamoeba histolytica and Entamoeba chattoni in Primate survey. J. Protozool. 29: 136-139.

Scott, M.E. 1988. The impact of infection and disease on animal populations: implications for conservation biology. Conserv. Biol. 2: 40-56.

Stoner, K.E. 1996. Prevalence and intensity of intestinal parasites in mantled howling monkeys (Alouatta palliata) in northeastern Costa Rica: implications for conservation biology. Conserv. Biol. 10-2: 539-546.

Stuart, M.D., L.L. Greenspan, K.E. Glander \& M.R. Clarke. 1990. Coprological survey of parasites of wild mantled howling monkeys, Alouatta palliata palliata. J. Wildlife Dis. 26: 547-549.

Stuart, M.D., V. Pendergast, S. Rumfelt, S. Pierberg, L. Greenspan, K.E. Glander \& M.R. Clarke. 1998. Parasites of wild howlers (Alouatta spp.). Int. J. Primatol. 19: 493-512.

Villalobos, F., A.A. Valerio \& A.P. Retana. 2004. A phylogeny of howler monkeys (Cebidae: Alouatta) based on 
mitochondrial, chromosomal and morphological data. Rev. Biol. Trop. 52: 665-677.

Zaldívar, M.E., O. Rocha, K.E. Glander, G. Aguilar, A.S. Huertas, R. Sánchez \& G. Wong. 2004. Distribution, ecology, life history, genetic variation, and risk of extinction of nonhuman primates from Costa Rica. Rev. Biol. Trop. 52: 679-693.

\section{REFERENCIAS DE INTERNET}

Swan, G.E. 1992. A summary of the practical aspects of drugs commonly used for the restraint of wild animals. Capture and Care Manual online; http://www. wildlifedecisionsupport.com/captureandcare/sectiona/drugs/01_swan.htm (consultado enero 2005). 
